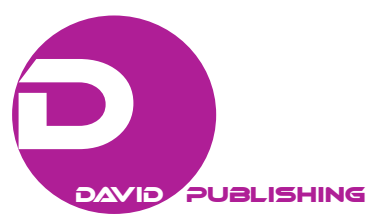

\title{
Towards Global Goal Orientation, Robustness and Integrity of Distributed Dynamic Systems
}

\author{
Peter Simon Sapaty \\ National Academy of Sciences of Ukraine, Kiev, Ukraine
}

\begin{abstract}
In an addition to the variety of topics in aims and scope of the Journal of International Relations and Diplomacy, we would be happy to offer here some more areas in line with its general orientation, which could be worth investigating, encouraging more paper submissions. These relate to effective organization of large distributed dynamic systems of diverse natures which may require innovative solutions reflecting the growing world dynamics in the 21 st century with emerging challenges and threats to local and global prosperity, stability, and safety.
\end{abstract}

Keywords: international relations, world dynamics, emerging challenges, distributed systems, goal orientation, integrity, interoperability, over-operability

\section{Introduction}

We are witnessing a dramatic change in the character of national and international activity, especially in crisis and conflict areas, with the use of asymmetric, unconventional, and hybrid solutions. They may simultaneously involve economy, ecology, international relations, ethnicity, culture, law, religion, etc., defense and military too, occupying both physical and virtual environments. And these solutions may need to be multidimensional and highly integral in order to succeed.

The management of national and international solutions is usually based on the concept of "interoperability" (Interoperability ${ }^{2}$; Slater, 2012), which is the quality of a system with well understood and clear interfaces to work with other systems without restrictions. There may be different kinds of interoperability. "Syntactic interoperability" means that two or more systems are just capable to communicate with each other. "Semantic interoperability" supposes that beyond the ability to exchange information, different systems are capable of interpreting the exchanged information. "Cross-domain interoperability" occurs when different kinds of entities (which may be multiple social, organizational, political, legal, etc.) can work together for a common purpose. Interoperability is the key principle for joint operations in both civil and military areas. For example, NATO has been based on interoperability since 1949 when it was founded. Interoperability allows organisations of different nationalities and armed services to conduct joint peacekeeping operations. It is also dominant in any international relief missions acting after natural or manmade disasters.

In this editorial note, we will touch insufficiency of the existing interoperability principles to organize

Peter Simon Sapaty, Chief Scientist, Institute of Mathematical Machines and Systems, National Academy of Sciences of Ukraine.

1 NATO Interoperability. Retrieved from

http://www.nato.int/nato_static/assets/pdf/pdf_publications/20120116_interoperability-en.pdf

${ }^{2}$ Retrieved from https://en.wikipedia.org/wiki/Interoperability 
solutions of complex multidimensional and hybrid problems, especially in highly dynamic and critical situations, highlighting the necessity of much more integral and holistic models and methods of organization of modern systems, whether technical, social, or combined. We will go back into the history and lift some fundamental system theories which along with modern trends and ideas may help to develop radically new system concepts for solving urgent problems of this century. We also provide a summary of our own approach being developed for decades and in different countries, which can believably be, similar to others, on a right way to the future. And encouragement of more paper submissions to this journal concludes our note.

\section{The Insufficiency of Interoperability Organizations}

The increased complexity of operations due to the growing world dynamics and emerging instability makes existing interoperability principles and organizations "not fully sufficient to provide the needed overall awareness, integrity, and pursuit of global goals" with runtime adjustment to new ones. The situations are often complicated by the necessity to operate in cyber-contested multi-dimensional spaces with high connectivity and inter-dependence, also with numerous actors having their own, often quite different purposes and interconnections. We are regularly witnessing failures of interoperability-based systems on international levels, where seemingly honest and noble intensions and actions of different players to improve and heal complex situations in certain places on the globe lead to quite unexpected results. As a matter of fact, this occurs "by not grasping properly the current and future entirety of the problem", and especially by the lack of adequate scientific and technological means for its expression and management.

On the way to increase integrity and wholeness of distributed systems and missions we may already have a useful background and support from known system theories and approaches, which are considering complex systems as "holistic organizations" rather than the collection of communicating parts defined independently. A brief description of some of them follows.

\section{Some Existing System Theories and Approaches}

\section{System Dynamics}

Fundamental works were carried out by Jay Forrester on analysis of complex systems (urban, industrial, world as a whole) with their detailed computer simulation (Forrester, 1961, 1969, 1971, 2009). It became clear that complex systems are "counterintuitive", where corrective actions in them are often ineffective or even adverse in the results. In complex systems the cause and the effect are often not closely related in either time or space, and their structure is not a simple feedback loop. In reality, complex system has a "multiplicity of interacting feedback loops", and its internal rates of flow are controlled by "nonlinear relationships". Complex systems are usually of high order, with many system states or levels. They may contain positive-feedback loops reflecting growth, and negative, goal-seeking ones. In the complex system the cause may lie far back and away from symptoms, and the causes are usually found not in prior events but rather "in the structure and policies of the system".

\section{Biology-Related General Systems Theory}

This theory after Ludwig von Bertalanffy $(1968,1950,1951)$ is based on similar general conceptions and viewpoints which have emerged and evolved in various disciplines. In the past, science tried to explain observable phenomena by reducing them to communication and interplay of elementary units which are independent from each other. New approaches are based on what is termed "wholeness", where "systems of 
various orders cannot be understood, even in principle, by investigation of their parts in isolation". Conceptions of this nature have appeared in all branches of science, regardless of which types of objects are being studied (i.e. inanimate things, living organisms, social phenomena, etc.).

\section{Gestalt Psychology}

"Gestalt"3 is both philosophy and psychology term which means "unified whole" (Wagemans et al., 2012; Wertheimer, 1922; Köhler, 1920; Koffka, 1935). It refers to visual perception theories developed by German psychologists at the beginning of the past century. These theories tried to understand and describe how people can organize visual elements into groups or "unified wholes" under certain conditions and principles, maintaining our meaningful perceptions in an apparently chaotic world. Forming a global whole with self-organizing tendencies is being the central principle of gestalt psychology, where "the whole has a reality of its own, independent of the parts", and our brain can generate whole forms, visually recognizing global figures instead of just collections of simpler and unrelated elements (like points, lines, curves, etc.).

\section{Integral Psychology}

The "integral psychology" (Wilber, 2000, 2003) is determined to embrace and unite all aspects of human consciousness under one concept, integrating ideas and models of consciousness, psychology, and therapy. The resultant psychological model includes "waves of development, streams of development, states of consciousness", and the "self". Under existing general consensus, neither mind nor brain can be reduced without each other, which means that both mind and brain need to be included in a "non-reductionistic way" in any integral theory of consciousness.

\section{Memetics}

"Memetics"4 (Dawkins, 1976; Hancock, 2010) is the theory of mental content based on an analogy with Darwinian evolution. Being an emerging subfield of psychology, memetics is considered as an approach to evolutionary models of cultural information transfer. The "meme", analogous to a "gene", was conceived as a "unit of culture" (like idea, belief, pattern of behaviour, etc.) which is "hosted" in the minds of one or more individuals, and which can reproduce itself, jumping from mind to mind. Memes spread through the social body similar to how genes spread through the biological body, forming the "invisible DNA of human society". Much like a virus moves from body to body, memes move from mind to mind. Memetics can be effectively used to identify and target specific root causes of challenging social problems in different areas; "military memetics" is being developed as its branch too (Finkelstein, 2011).

\section{Human Terrain}

Whereas geographic terrain, or merely "terrain", is used to represent vertical and horizontal dimensions of land surface, "human terrain" (Gonzalez, 2008; Jacob et al., 2006; Joint Doctrine, 2013; Sapaty, 2014) deals with human population, its culture and interactions, being a new and rapidly growing field of research originated, as a term, from military operations. All local and global conflicts are about people: their behaviors, attitudes, fears, social structures, family and ideological ties and narratives. Understanding the human dimensions of conflict is therefore a "critical determinant in preventing conflicts", shaping them and influencing the actors involved. It contributes to strategic awareness, ability to plan and execute operations,

\footnotetext{
${ }^{3}$ Gestalt Psychology. Retrieved from https://en.wikipedia.org/wiki/Gestalt_psychology

${ }^{4}$ Retrieved from https://en.wikipedia.org/wiki/Memetics
} 
helps to identify threats and opportunities. Human terrain is defined as "characterizing cultural, anthropological, and ethnographic information about the human population" and interactions within the operations area. Human terrain analysis is the process through which understanding of the human terrain is developed. It integrates "human geography and cultural information".

\section{Our Own Contribution to the Area}

We have developed an approach called "over-operability" (Sapaty, 2002-2015) in both opposition and extension to the currently dominant interoperability, which allows for integral global-goal-driven solutions in distributed environments. This approach uses creatively the ideas briefed in the previous section. The resultant Spatial Grasp Technology (SGT) with Spatial Grasp Language (SGL) as its key element has been prototyped and tested on numerous researched applications. In most general terms it operates as symbolically shown in Figure 1.

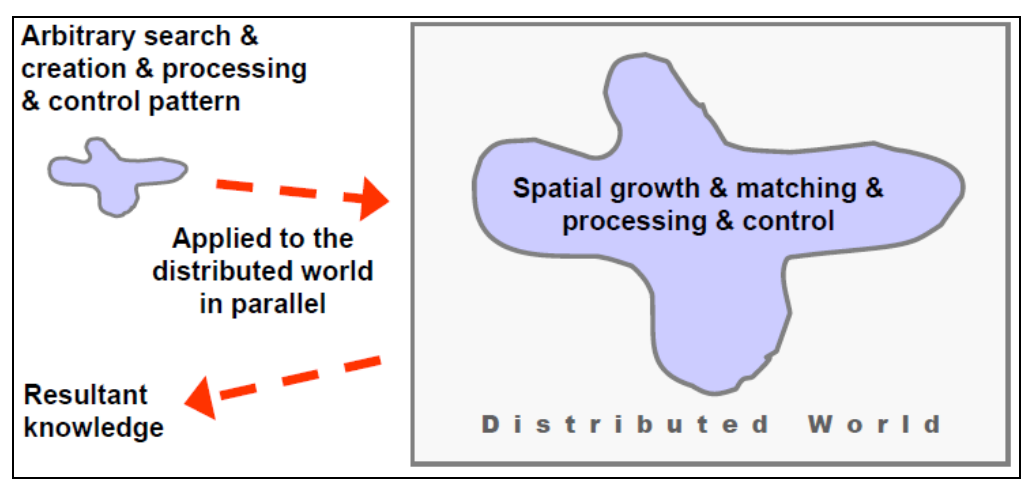

Figure 1. SGT main idea.

A high-level scenario for any task to be performed in a distributed world is represented as an "active self-evolving pattern" rather than traditional program, sequential or parallel. This pattern, expressing top semantics and key decisions of the problem to be solved, being applied in one or a number of world points (simultaneously or at different moments of time), "spatially grows, covers, and matches" the world. It can create, control, and change parts of the world or its whole, with final results retained in the distributed spaces (generally as active self-organized distributed infrastructures) or returned to the starting point (or points) as detailed data or high level knowledge, whatever required. The organization and evolution of this matching pattern is based on "parallel wavelike" coverage, or "grasping" of distributed physical and virtual spaces, as symbolically shown in Figure 2, which can be supported by a strong feedback control and return of remote results.

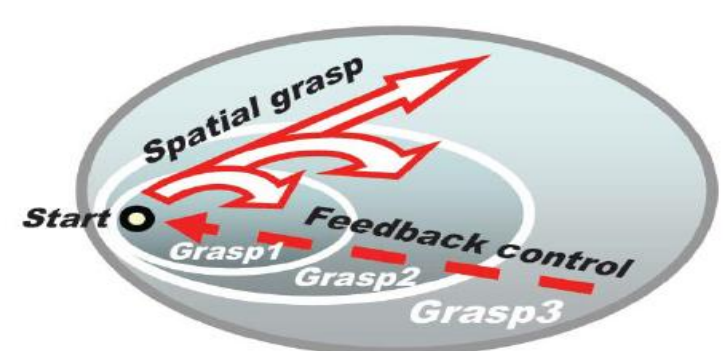

(a)

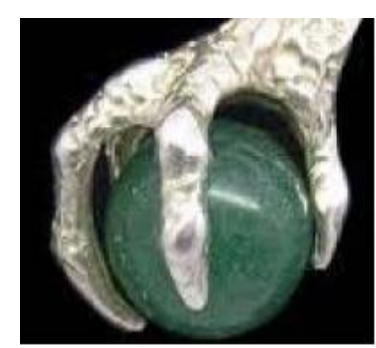

(b)

Figure 2. The matching pattern's evolution: (a) Controlled wavelike grasping of distributed worlds; (b) Symbolic physical analogy. 
The matching pattern evolves as a "succession of grasps", each potentially developing in parallel from the final virtual or physical points reached by the previous grasps. Invocation of new grasps may be right after reaching final results by the previous grasps, or may need synchronization and waiting for the completion of all (or parts of) the previous grasps. The body of the matching pattern can "replicate and freely propagate" in space during its evolution, with "utilized parts omitted" if not needed any more. The matching pattern can also start from multiple sources and by different authorities, as shown in Figure 3, with constituent patterns "cooperating or competing" in the distributed space, or being independent from each other.

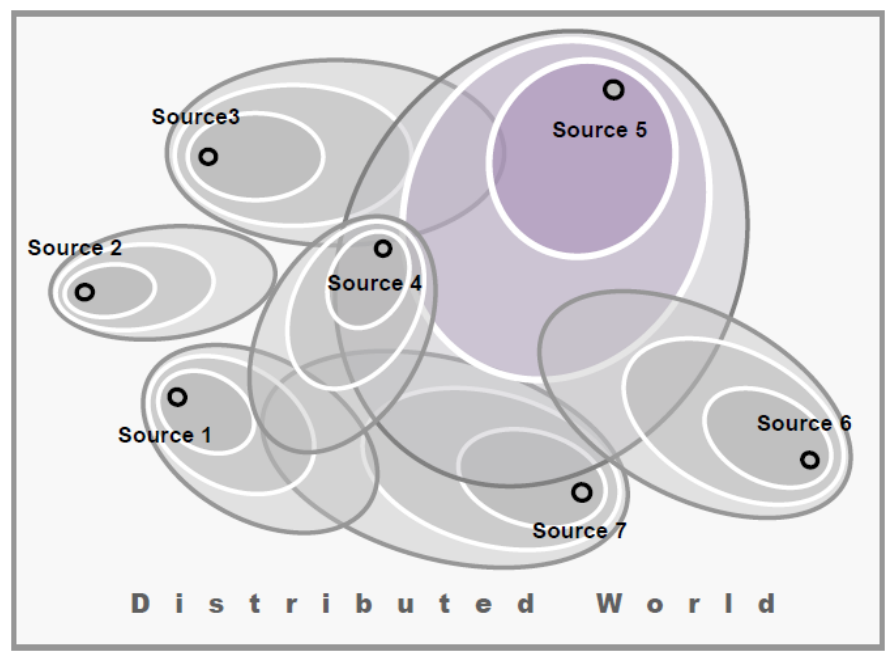

Figure 3. Multisource world coverage \& matching.

SGL, describing these matching patterns, directly operates with "physical, virtual" and "executive" words, as well as their combinations. It has a "universal recursive structure" (see Figure 4), which allows us to express any spatial algorithms, create and manage any distributed structures and systems, static or dynamic, passive or active, solving any problems in, on, and over them, and this can be expressed in a compact and unified way.

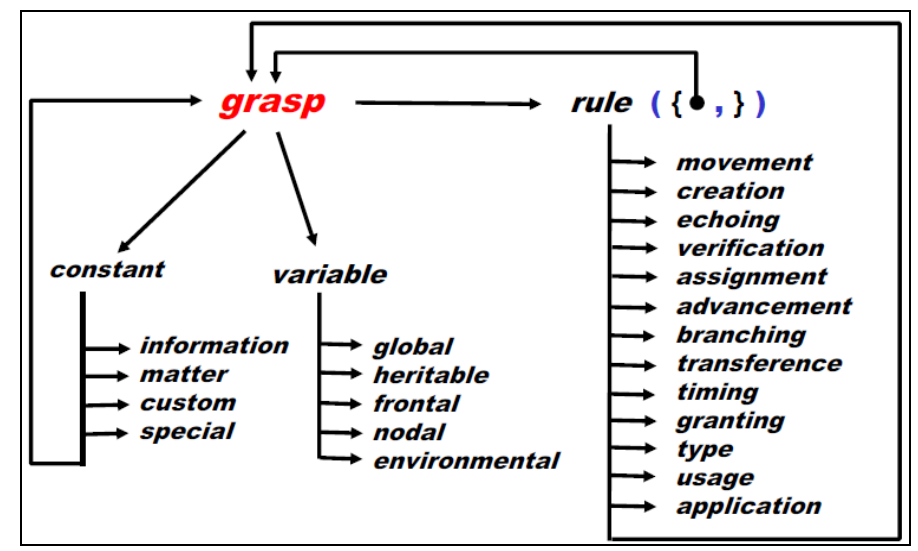

Figure 4. SGL universal recursive syntax.

SGT in practice operates as shown in Figure 5. A distributed network of SGL interpreters (as universal control modules $\mathrm{U}$ ) embedded into key system points (humans, robots, smart sensors, mobile phones, internet hosts, laptops, etc.) "collectively interprets high-level mission scenarios" written in SGL. This effectively converts the whole society into a "universal spatial supercomputer" (more correctly: super-machine, as it can 
operate with physical matter too, also move in parts or as a whole in physical spaces) on, and by which, any optimization and management tasks can be programmed and solved in distributed societies.

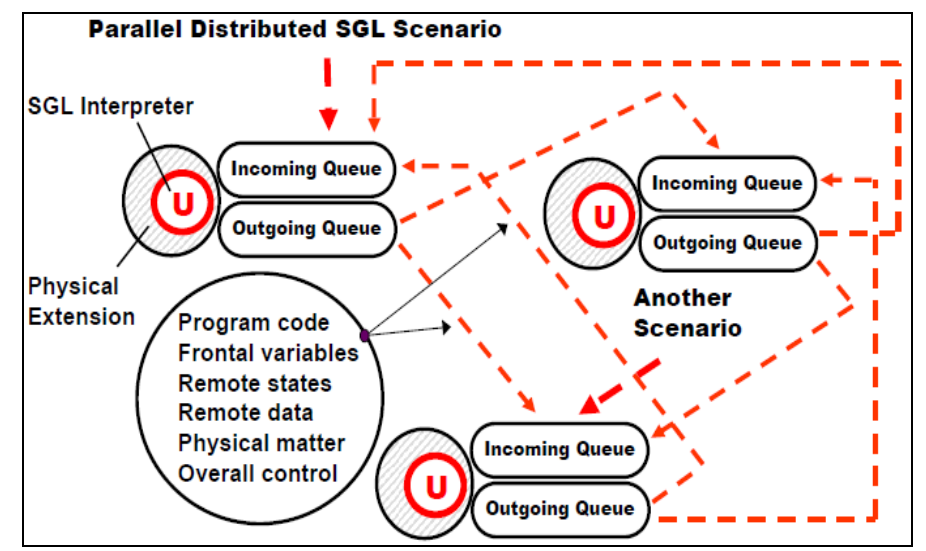

Figure 5. SGL interpretation network as a universal parallel spatial machine.

The spreading scenarios create "knowledge infrastructures" arbitrarily distributed between system components (see Figure 6). Navigated by same or other scenarios, these can effectively support distributed databases, command and control, situation awareness and autonomous decisions, also "simulate any other existing or hypothetical" computational and/or control models.

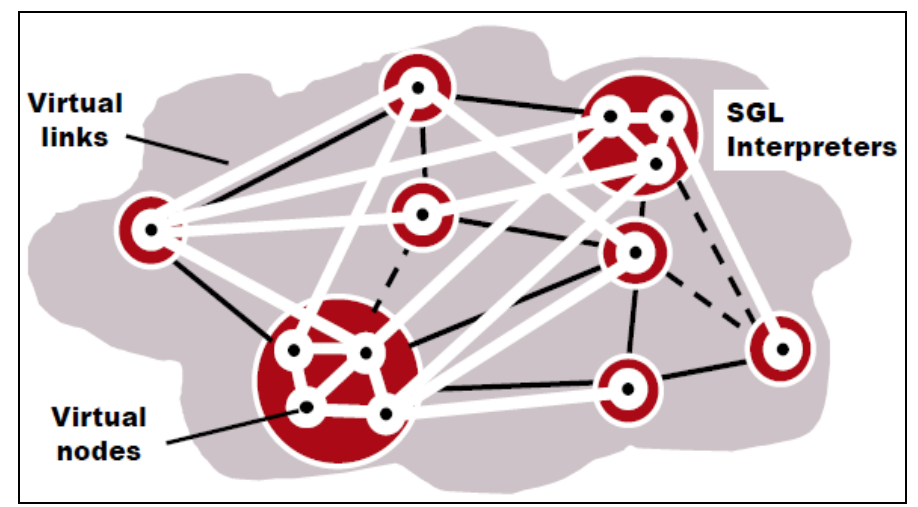

Figure 6. Creating spatial infrastructures by SGT.

The technology developed has been investigated and used to find integral solutions in distributed dynamic systems, including those related to collective behavior of large numbers of cooperating robotic units, critical infrastructure investigation, creation and protection, automated up to fully automatic command and control, advanced maritime operations, spatial intelligence, missile defense, night vision, finding certain structures in social systems, and many others. More on this can be found in the existing publications including the recent ones (Sapaty, 2015-2016), in the current journal too (Sapaty, 2014), also in earlier books (Sapaty, 1999, 2005).

SGT allows us to describe and implement "integral spatial solutions" on a variety of levels and their mixtures, including topmost semantic ones, where high-level mission scenarios reflecting main operations and decisions can always survive, with particular system components, their numbers and interactions, also overall management and control being clarified and updated at runtime, thus "automatically adjusting to the rapidly changing goals and environments". 


\section{Conclusions}

New ideas, models, methods and research \& implementation results on the wholeness and integrity of large distributed dynamic systems, which may lead to advanced solutions of most critical problems in the 21 st century, are very much welcome at the Journal of International Relations and Diplomacy. These being of great need in numerous areas related to (just named only a few): economy, ecology, international relations, demographics, education, infrastructure protection, security, crisis management, and defense. We will be happy to provide any needed encouragement and support for their development, detailed assessment, implementation, international networking, and quick publication.

\section{References}

Bertalanffy, L. von. (1968). General system theory foundations, development, applications. New York : George Braziller. Bertalanffy, L. von. (J1950). The theory of open systems in Physics and Biology. Science, 111(2872), 23-29. .

Bertalanffy, L. von (1951). Theoretical models in Biology and Psychology. Journal of Personality, 20(1), 24-38.

Dawkins, R. (1976). The selfish gene. Oxford University Press.

Finkelstein, R. (2011). Tutorial: Military memetics. Robotic Technology Inc. Retrieved from http://www.robotictechnologyinc.com/

Forrester, J. (1969). Urban dynamics. Pegasus Communications.

Forrester, J. (1971). World dynamics. Wright-Allen Press.

Forrester, J. (1961). Industrial dynamics. Waltham, MA: Pegasus Communications.

Forrester, J. (2009). Learning through system dynamics as preparation for the 21st century. Retrieved from http://clexchange.org/ftp/documents/whyk12sd/Y_2009-02LearningThroughSD.pdf

Gonzalez, R. (February 2008). Human terrain: Past, present and future applications. Anthropology Today, 24(1), 21-26.

Hancock, B. J. (April 2010). Memetic warfare: The future of war. Military Intelligence, PB 34-10-2 , 36(2), 41-46.

Jacob, K., Lester, G. L., Prinslow, K., \& Smith, D. (2006, October). The human terrain system: A CORDS for the 21 st century. Defense Technology Information Center (DTIC).

Joint doctrine note 4/13, Culture and Human Terrain. (2013, September). Retrieved from https://www.gov.uk/government/publications/culture-and-human-terrain

Köhler, W. (1920). Die physischen Gestalten in Ruhe und im stationären Zustand. Eine natur-philosophische Untersuchung. Braunschweig. Germany: Friedr. Vieweg und Sohn.

Koffka, K. (1935). Principles of Gestalt psychology. London, UK: Lund Humphries.

Slater T. (2012). What is interoperability? Network Centric Operations Industry Consortium-NCOIC.

Sapaty, P. (1999). Mobile processing in distributed and open environments. John Wiley \& Sons, New York.

Sapaty, P. (2002). Over-operability in distributed simulation and control. The MSIAC's M\&S Journal Online, 4(2), 8.

Sapaty, P. (2005). Ruling distributed dynamic worlds. John Wiley \& Sons, New York.

Sapaty, P. (2008). Grasping the whole by spatial intelligence: A higher level for distributed avionics. Proc. international conference Military Avionics 2008, Jan. 30 - Feb.1, Café Royal, London, UK.

Sapaty, P. (2009). Gestalt-based integrity of distributed networked systems. SPIE Europe Security + Defence, bcc Berliner Congress Centre, Berlin Germany.

Sapaty, P. (September 2014). Distributed human terrain operations for solving national and international problems. International Relations and Diplomacy, 2(9), 597-622.

Sapaty, P. (October 2015). Providing over-operability of advanced ISR systems by a high-level networking technology. SMI's Airborne ISR, Holiday Inn Kensington Forum, London, United Kingdom.

Sapaty, P. (2015). Military robotics: Latest trends and spatial grasp solutions. International Journal of Advanced Research in Artificial Intelligence, 4(4). Retrieved from http://thesai.org/Publications/IJARAI

Sapaty, P. (2016a). Spatial grasp language (SGL). Advances in image and video processing, 4(1). Retrieved from http://scholarpublishing.org/index.php/AIVP/

Sapaty, P. (2016b). Towards massively robotized systems under spatial grasp technology. Journal of Computer Science \& Systems Biology, 9(1). Retrieved from http://www.omicsonline.org/computer-science-systems-biology.php 
Sapaty, P. (2016c). A brief introduction to the spatial grasp language (SGL). Journal of Computer Science \& Systems Biology, 9(2). Retrieved from http://www.omicsonline.org/computer-science-systems-biology.php

Sapaty, P. (2016d). Providing integrity of distributed ISR systems under spatial grasp paradigm. Journal of Engineering Sciences and Research Technology, 2(1). Retrieved from http://sansgroup.org/journal.php?journal=52

Sapaty, P. (2016e). Advanced maritime operations under network-centric organizations. Proc. International Conference Warship 2016: Advanced Technologies In Naval Design \& Construction \& Operation, 15-16 June 2016, Guildhall, Bath, UK. Retrieved from http://www.rina.org.uk/Warship2016

Wagemans, J., Elder, J. H., Kubovy, M., Palmer, S. E., Peterson, M. A., Singh, M., \& Heydt, R. von der (2012). A century of gestalt psychology in visual perception: I. Perceptual Grouping and Figure-Ground Organization. Psychological Bulletin, $138(6), 1172$.

Wertheimer, M. (1922). Untersuchungen zur Lehre von der Gestalt, I: Prinzipielle Bemerkungen. Psychologische Forschung.

Wilber, K. (2000). Integral psychology: Consciousness, spirit, psychology, therapy. Shambala Publications, Boston.

Wilber, K. (2003). Waves, streams, states, and self: An outline of an integral psychology. The Humanistic Psychologist, 31(2-3), $22-49$. 